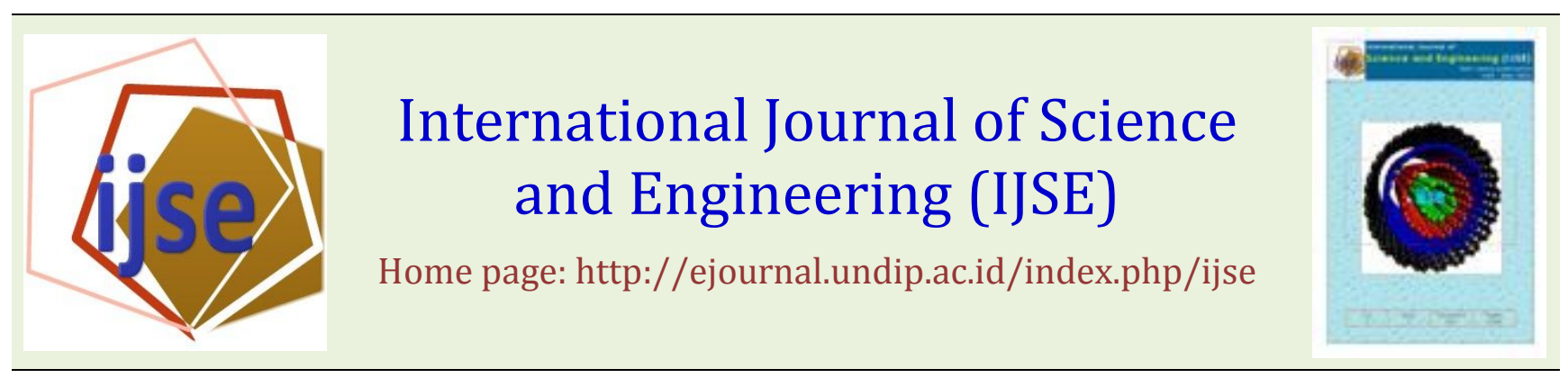

\title{
Study of Biometric Identification Method Based on Naked Footprint
}

\author{
Raji Rafiu King \#1, Wang Xiaopeng ${ }^{* 2}$ \\ \# School of Electronic and Information Engineering, Lanzhou Jiaotong University, \\ 88 West Anning Road, Lanzhou, 730070, China \\ ${ }^{1}$ ralphigwedyahoo.com \\ ${ }^{2}$ Wangxp1969@sina.com
}

\begin{abstract}
The scale of deployment of biometric identity-verification systems has recently seen an enormous increase owing to the need for more secure and reliable way of identifying people. Footprint identification which can be defined as the measurement of footprint features for recognizing the identity of a user has surfaced recently. This study is based on a biometric personal identification method using static footprint features viz. friction ridge / texture and foot shape / silhouette. To begin with, naked footprints of users are captured; images then undergo pre processing followed by the extraction of two features; shape using Gradient Vector Flow ( GVF) snake model and minutiae extraction respectively. Matching is then effected based on these two features followed by a fusion of these two results for either a reject or accept decision. Our shape matching feature is based on cosine similarity while the texture one is based on miniature score matching. The results from our research establish that the naked footprint is a credible biometric feature as two barefoot impressions of an individual match perfectly while that of two different persons shows a great deal of dissimilarity.
\end{abstract}

Keywords - biometric; footprint; friction ridge; silhouette; verification

Submission : August 1, $2013 \quad$ Corrected : September 10, 2013

Accepted: September 21, 2013

Doi: $10.12777 /$ ijse.5.2.29-35

How to cite this article: King, R.R. and Xiaopeng, W. (2013). Study of Biometric Identification Method Based on Naked Footprint . International Journal of Science and Engineering, 5(2),18-24. Doi: 10.12777/ijse.5.2.29-35]

\section{INTRODUCTION}

The persistent insecurity in our world today has necessitated the continuous search for new forms of security which are more reliable and less vulnerable against intruders' attacks. Direct attacks are carried out by falsifying the biometric trait of a user and then presenting this falsified information to the biometric system to gain access. One such example [11] is to fool a fingerprint system by copying the fingerprint of another person and creating an artificial or gummy finger which can then be presented to the biometric system to falsely gain access. It is almost impossible to obtain a person's footprint without his consent. We are therefore considering a technique that will minimize these rather grave instances of stolen biometric traits as much as possible hence the choice of footprint

Every part of the human body is unique in itself and it is amazing to learn that every part of the body is different in its own way from a similar part in another person's body. Biometrics which is the use of biological or behavioural features of an individual to determine his identity is based on this premise. Personal identification using footprint can be carried out by using any one of the two important features of the foot; namely static and dynamic.

Static feature requires stand-up posture at fixed positions every time from the subject, whereas the dynamic feature deals with the walking behaviour of a person. This proposed system however is based on the former. Humans usually have five toes on each foot. Exceptions include polydactyl (more than five toes), and syndactyly or amputation (fewer than five toes).

Forefoot shape, including toe shape, exhibits significant variation among people. Hawes et al [4] contends that specifically measuring toe and forefoot metrics for humans include: height, breadth and girth, ball length, digital pattern, heel to toe length and footprint layout. The shape and size features of the foot, defined as the morphology of the foot are extracted and classified based on shape, and minutiae. A look at a couple of recently proposed footprint recognition systems included those that make use of several features such as normalized 
Static footprint based on Euclidean distance[7], Modified Sequential Haar Energy Transform (MSHET) [1], Center of pressure (COP) Using Principle Component Analysis (PCA) [2], Four orientation feature based approaches namely "Ordinal Code" (OC), "Binary Orientation Co-occurrence Vector" (BOCV), "Competitive Code" (CC), and "Robust Line Orientation Code" (RLOC) [12], Hidden Markov Models (HMM) [5] are exploited for recognition.

\section{PROPOSED METHOD}

In this paper a foot print verification is implemented using static footprint features. In this method two distinct features such as shape and minutiae (also referring to the texture) are respectively extracted. Matching is done to arrive at a reject or an accept decision.

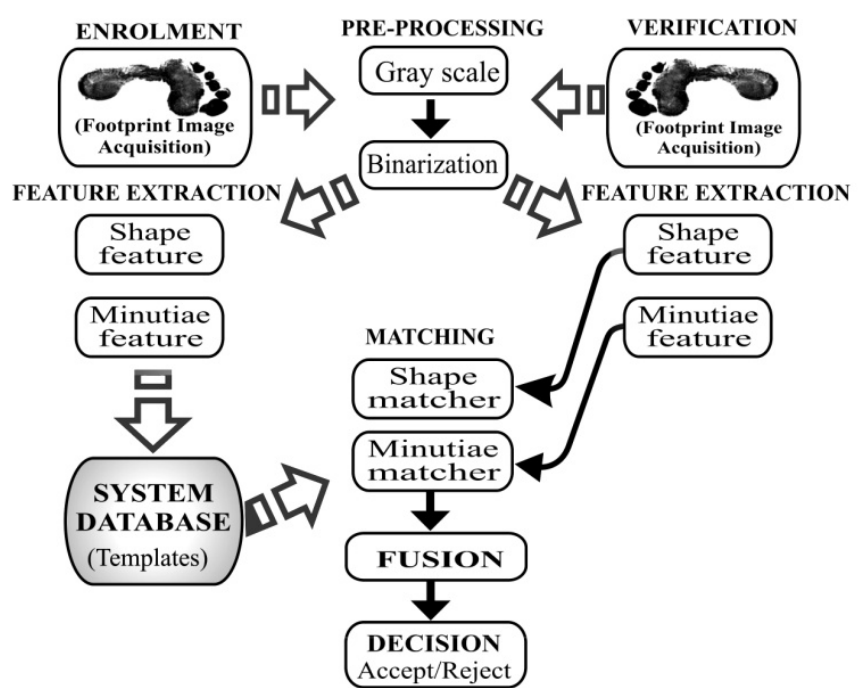

Figure 1: Proposed footprint identification system

The proposed technique operates by acquiring biometric information from an end user. This is followed by the processing and extraction of specified features from the sample and then saving the extracted information in a database, subsequently referred to as enrolment.

This is followed by equating newly acquired biometric information with the one(s) contained in the database. Deciding how well they match and indicating whether or not an identification or verification has been achieved ends the process.

\section{A. Enrolment}

Static Footprint images were captured via a Canon Scan Lide110 scanner from volunteers. Each user in a standing posture submits his/her footprint image.

\section{B. Preprocessing}

Captured images undergo preprocessing. The coloured images are converted to gray scale and subsequently followed by binarization which involves assigning a threshold value. However due to the fact that this study employed two distinctive feature extraction processes, so were the preprocessing methods for the respective feature extraction processes.

\section{Feature Extraction}

\section{1) Shape/silhouette feature:}

In order to ensure performance optimization of a biometric system, the system must include as many elements as possible hence the choice of geometry and minutia cues for identification. Geometric measurements are frequently used in biometric systems due to their simplicity, robustness to injuries and high collectability. They usually don't require highly-resolved binary images.

Snakes [3] - defined as energy-minimizing curves that change in form to fit image features have been used in this paper. They are frequently applied in computer vision and image analysis to recognize and map objects, and to define their shape. Snakes are described as curves specified within an image region that can actuate under the manipulation of internal forces inherent in the curve itself and external forces deduced from image data.

The classical snake algorithms are plagued with two main drawbacks. First, the initial contour must, generally, be near to the true boundary or otherwise it potentially might merge producing undesirable results. The next limitation is that, it is not easy for the active contour to advance into concave boundary regions. "Gradient Vector Flow snake" (GVF), espoused by Chenyang Xu et al [3] had the solution to the above challenges enumerated hence it being the preferred snake for this study. A traditional snake is a curve $X(s)=[x(s), y(s)], s \in[0,1]$ that actuates through the spatial domain of an image $f(x, y)$ to minimize the energy function, where $x(s)$ and $y(s)$ are $x$, $y$ coordinates along the contour and $s$ denotes the length of arc with values in $[0,1]$. The energy functional to be minimized is expressed as:

$$
E_{\text {energy }}=\int_{0}^{1} E_{\text {in }}(X(s))+E_{\text {ext }}(X(s)) d s
$$

Where $E_{\text {in }}=\frac{1}{2}\left[\alpha\left|X^{\prime}(s)\right|^{2}+\beta\left|X^{\prime \prime}(s)\right|^{2}\right]$ is the internal energy that renders smoothness to the contour, $\alpha$ and $\beta$ are weighting parameters that regulate the snake's tension and rigidity. $E_{\text {ext }}$ is the image energy computed from the image data and it presses the snake towards the required objects. A final solution will be obtained by the minimum total snake energy which must satisfy the Euler equation:

$$
\alpha X^{\prime \prime}(s, t)-\beta X^{\prime \prime "}(s, t)-\nabla E_{\text {ext }}=0
$$

Then the snake is made dynamic by treating as function of time the following:

$$
X_{t}^{\prime \prime}(s, t)-\alpha X^{\prime \prime}(s, t)-\beta X^{\prime \prime \prime}(s, t)-\nabla E_{e x t}
$$

The term $X_{t}(x, t)$ is eliminated when the solution $X(x, t)$ stabilizes. The force-balance equation can therefore be seen as:

$$
F_{\text {in }}+F_{\text {ext }}{ }^{(p)}=0
$$

Where $F_{i n}=\alpha X^{\prime \prime}(s)-\beta X^{\prime \prime "}(s)$ is the internal force that attempts to truncate the expansion and bending and 
$F_{e x t}{ }^{(p)}=\Delta E_{e x t}$ is the external potential that tries to draw snake towards the desired image edge. Using the force balance condition as an initial point, a new nonirrotational external force field, referred to as GVF field is defined. The GVF field is the dense vector field obtained from the test image by minimizing energy function in a mutational framework. The GVF field replaces the external force field. The GVF field has been defined as:

$$
\mathrm{V}(\mathrm{x}, \mathrm{y})=[\mathrm{u}(\mathrm{x}, \mathrm{y}), \mathrm{v}(\mathrm{x}, \mathrm{y})]
$$

This minimizes the energy function in equation (1)

$$
\varepsilon=\iint \mu\left(u_{x}^{2}+u_{y}^{2}+v_{x}^{2}+v_{y}^{2}\right)+|\nabla f|^{2}|v-\nabla f|^{2} d x d y
$$

Where $f$ is the edge map which is derived from using an edge detector on the original image convoluted with a Gaussian kernel, and is a regularization parameter controlling the tradeoff between the first term and the second term in the integrand.

The external field ensuing from this minimization can be anticipated to be either entirely irrotational (as potential field of the classical snake) or entirely solenoidal. Applying calculus variations, we can obtain the GVF by solving the following Euler equations:

$$
\begin{gathered}
\mu \nabla^{2} u-(u-f)_{x} \cdot\left(f_{x}{ }^{2}+f_{y}{ }^{2}\right)=0 \\
\mu \nabla^{2} v-(v-f x) \cdot\left(f x^{2}+f y^{2}\right)=0
\end{gathered}
$$

In the Euler equation the smooth areas; $f x$ and $f y$ are zeros and so the vector field $u(x, y)$ and $v(x, y)$ is expressed by the first term. The Laplacian operator is denoted by $\nabla^{2}$, we then solved Equations (6a) and (6b) by dealing with $u$ and $v$ as functions of time $t$ and for convenience is expressed as:

$$
\begin{gathered}
u_{t}(x, y, t)=\mu \nabla^{2} u(x, y, t)-b(x, y)\left(u(x, y, t)+c^{1}(x, y)\right. \\
v_{t}(x, y, t)=\mu \nabla^{2} v(x, y, t)-b(x, y) v(x, y, t)+c^{2}(x, y)
\end{gathered}
$$

Where,

$$
\begin{aligned}
& b(x, y)=f_{x}(x, y)^{2}+f_{y}(x, y)^{2} \\
& c^{1}(x, y)=b(x, y) f_{x}(x, y) \\
& c^{2}(x, y)=b(x, y) f_{y}(x, y)
\end{aligned}
$$

After computing the GVF field, regard it as outside force and apply it to the iterative equation (2). Using iterative calculation, we can find the solutions when the time variable is arriving at a steady state. The peculiar strengths of GVF snake over a classical snake are its unresponsiveness to initialization and ability to advance into concave boundary regions.

\section{2) Minutiae feature:}

Typical ridge structures characteristic of fingerprints are also present in footprints at desirable resolutions even if no special ridge scanning devices are used. As a result, a texture-based feature extraction has been included and thus ridge details from the big toe of the foot are then extracted.

Several categories of ridge orientations have been established including the Henry system: whorl, right loop, left loop, arch, and tented arch etc. Our minutiae verification is based on the Minutia Score Matching method (MSM) [9]. The process includes binarization Thinning, Minutiae extraction, filtering, Minutiae matching and Computing matching score. Binarization of the image is effected. After this operation, ridges in the print are shown in black colour while the furrows are in white. Minutiae extraction operation deduces the minutiae location and the minutiae angles.

\section{Matching}

Template matching involves comparing the user template with templates from the database using a matching metric. The matching measurement gives a measure of affinity between two footprint templates. It generates a range of values when equating templates from the same footprint, and another range of values when equating templates from different footprints.

\section{1) Shape matching}

Our Foot shape matching was effected using cosine similarity [8] which is the measure of congruity between vectors of two images. Cosine Similarity is a standard measure used to equate two vectors by calculating the angular difference between them. The outcome of this calculation is ranked from ' 0 ' to ' 1 ', thus if it gets close to ' 1 '; it implies that the congruence of the two vectors is high or otherwise.

Expression of the equation is:

$$
\text { Similarity }=\cos (\theta)=\frac{A \cdot B}{\|A\|\|B\|}=\frac{\sum_{i=1}^{n} A_{i} \times B_{i}}{\sqrt{\sum_{i=1}^{n}\left(A_{i}\right)^{2}} \times \sqrt{\sum_{i=1}^{n}\left(B_{i}\right)^{2}}}
$$

Where $\theta$ stands for the angular between $A$ and $B$; the query image is represented by vector $A$ while the image in database is represented by vector $B$.

\section{2) Minutiae matching}

To compare the input foot print data with the template data, Minutiae matching are used. For efficient matching process, the extracted information is stored in the matrix format. The data matrix is as follows:

Number of rows: Number of minutiae points.

Number of columns: 4

Column 1: Row index of each minutia point.

Column 2: Column index of each minutia point.

Column 3: Orientation angle of each minutia point.

Column 4: Type of minutia. (A value of ' 1 ' is assigned for termination, and ' 3 ' is assigned for bifurcation).

Each input minutiae point is matched against a corresponding template minutiae point during the matching process. In each case, template and input minutiae are selected as reference points for their respective data sets. The reference points are used to convert the remaining data points to polar coordinates. 
The Equation (10) below [8] is used to convert the template minutiae from row and column indices to polar coordinates;

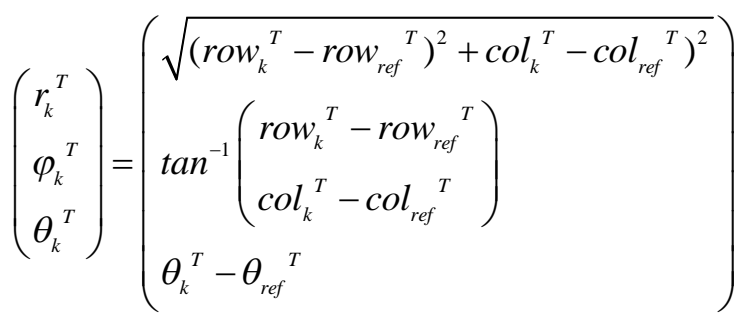

Where for a template image, $r_{k}^{T}=$ radial distance of $K^{\text {th }}$ minutiae, $\varphi_{k}{ }^{T}=$ radial angle of the minutiae, $\theta_{k}^{T}=$ orientation angle of the $K^{\text {th }}$ minutiae, $\operatorname{row}_{\text {ref }}{ }^{T} \cdot \operatorname{row}_{\text {ref }}{ }^{T}=$ row of index and column $K^{\text {th }}$ index of reference points. Similarly the input matrix data points are converted to polar coordinates using the Equation (11) below [8].

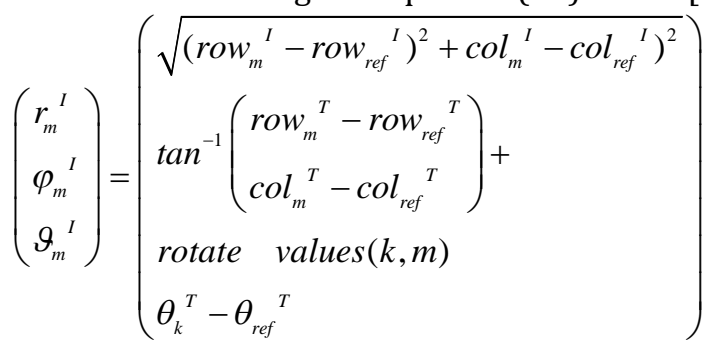

Rotate values $(k, m)$ denotes the difference between the orientation angles of $T k$ and $(I, m) . T k$ and $(I, m)$ denote the extracted data in all the columns of row $k$ and row $m$ in the template and input matrices, respectively.

\section{E. Fusion}

Biometric fusion is used to increase accuracy of a biometric system. When match scores output by different biometric matchers are consolidated in order to arrive at a final recognition decision. It must be noted that the match scores generated by the individual matchers may not be homogeneous hence the need for score normalization before subsequent fusion.

\section{1) Min-max normalization}

Score normalization refers to changing the location and scale parameters of the matching score distributions at the output of the individual matchers, so that the scores of different matchers are transformed into a common domain. Min-max normalization [10] is best suited for the case where the bounds (maximum and minimum values) of the scores produced by a matcher are known. In this case, we can easily transform the minimum and maximum scores to 0 and 1 , respectively. However, even if the match scores are not bounded, we can estimate the minimum and maximum values for the given set of training match scores and then apply the min-max normalization.

Let $s_{j}^{i}$ denote match score output by the $j^{\text {th }}$ matcher, $i=1,2 \ldots, N ; j=1,2 \ldots, R(R$ is the number of matchers and $N$ is the number of match scores available in the training set). The min-max normalized score, $n s_{j}^{t}$, for the test score $s_{j}^{t}$ is given by

$$
n s_{j}^{t} \frac{s_{j}^{t}-\min _{i=1}^{N} s_{j}^{t}}{\max _{i=1}^{N} s_{j}^{i}-\min _{i=1}^{N} s_{j}^{s}}
$$

Min-max normalization retains the original distribution of scores except for a scaling factor and transforms all the scores into a common range $[0,1]$. Distance scores can be transformed into similarity scores by subtracting the normalized score from 1.

2) Sum rule fusion

The procedure for sum rule-based fusion [6] is can be explained as follows. Based on a set of normalized scores $\left(x_{1}, \ldots . x_{m}\right)$ from a particular user (here the index $i=1, \ldots . m$ indicates the biometric matcher), the fused score $f s$ is evaluated using the formula:

$$
f s=w_{1} x_{1}+\ldots .+w_{m} x_{m}
$$

The notation $w_{1}$ stands for the weight which is assigned to the matcher $-i$, for $i=1, \ldots . m$. There are many choices of how to calculate these weights based on some preliminary results; a decision to use equal weights in our experiments was made. Then simplifying, it's expressed as:

$$
f s=x_{1}+\ldots .+x_{m}
$$

In the next step, the fused score $f s$ will be compared to a pre- specified threshold $t$. We declare a person to be genuine if $f s \geq t$, otherwise, we declare him/her an impostor.

\section{F. Decision}

Ultimately a determination of whether the user of the system is authentic or imposter is effected. The individual matching scores are aggregated to generate a single scalar score which is then used to make the final decision.

\section{EXPERIMENTS}

The experiment has been carried out using Matlab7.11.0.584 (R2010b) platform. We considered 16 users comprising Africans, Asians and Europeans, all university students of Lanzhou Jiaotong University aged between 20 and 30years. Each user presented four images; therefore the system database had a total of 64 images.

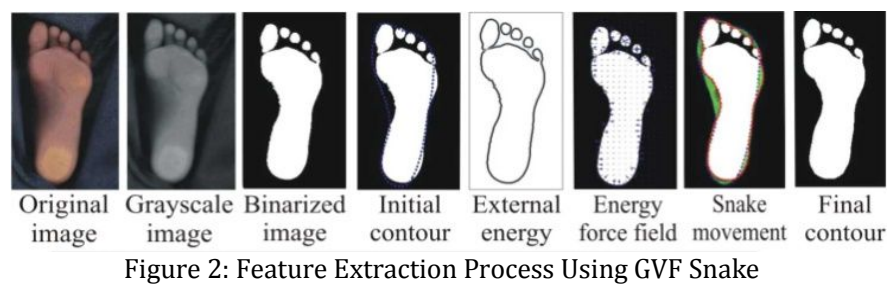

Our first feature (shape/silhouette) was extracted using GVF snake is shown above. 
The minutiae feature was extracted from the big toe of the user as shown above. User matching was done to arrive at the decision to determine if a user is genuine or an imposter. Below also shows the minutiae verification process.

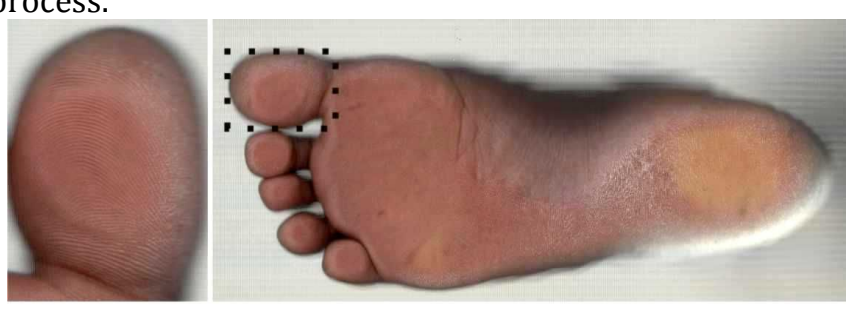

Figure 3: Minutiae Feature Extraction (big toe)

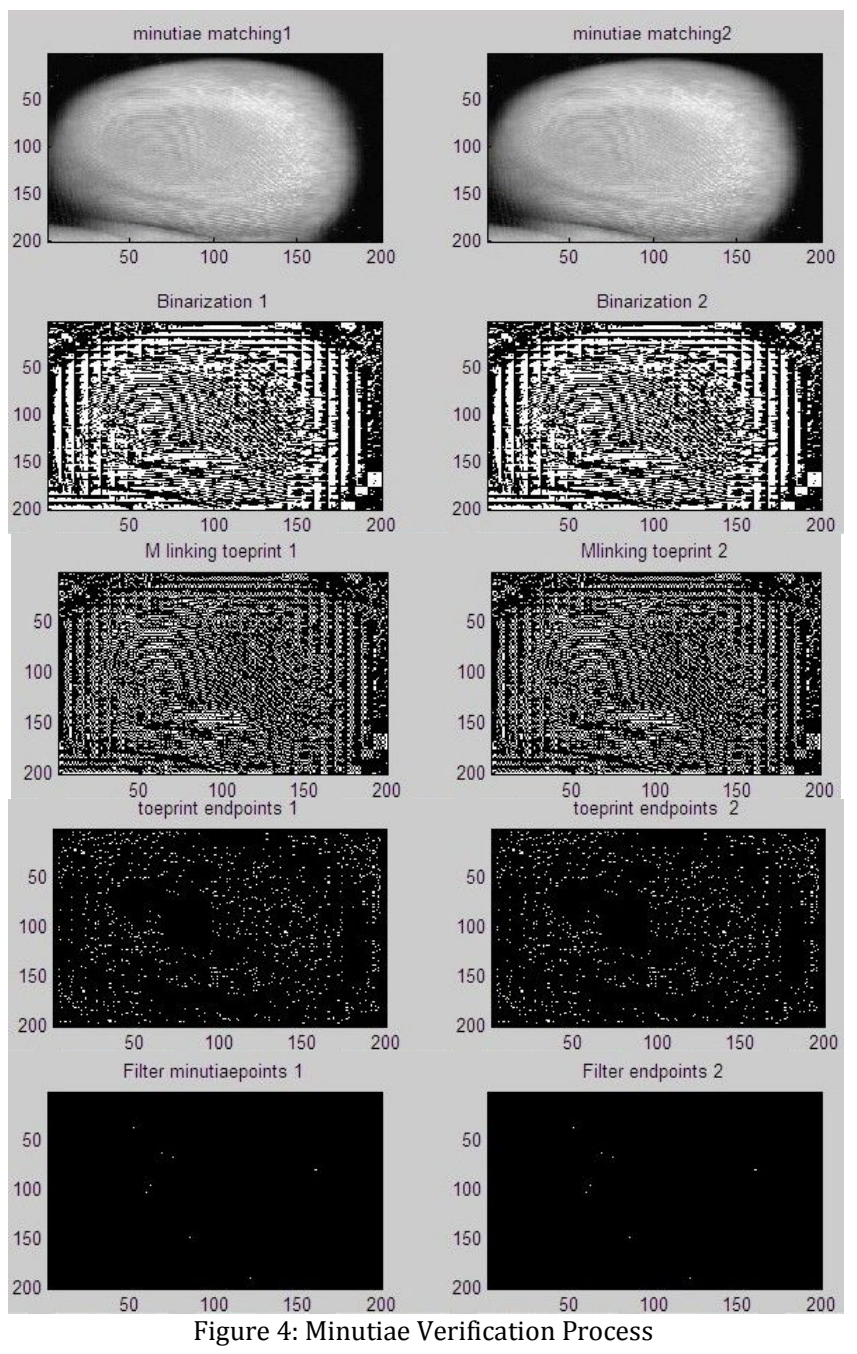

\section{IV.RESULTS AND DISCUSSIONS}

The above results show a high degree of accuracy with minutiae matching verification clocking 100\% accuracy. Imposter verification results yielded an average of $25 \%$. User verification was executed by comparing a user's data (footprint) with that in the system database.

Table 1 compares the verification accuracy of the proposed system with other footprint based biometric systems. As shown, our proposed system performed creditably well as compared with other footprint based techniques.
Table I: Recognition Accuracy Compared with Other Methods

\begin{tabular}{lccc}
\hline Method & Reference & $\begin{array}{c}\text { Size of } \\
\text { database }\end{array}$ & $\begin{array}{c}\text { Accuracy } \\
\%\end{array}$ \\
\hline $\begin{array}{l}\text { Proposed } \\
\text { method }\end{array}$ & 16 & 98.97 \\
$\begin{array}{l}\text { (HMM) } \\
\text { (MSHET) }\end{array}$ & $\begin{array}{c}\text { Jung et al. [7] } \\
\text { V.D.A Kumar } \\
\text { et al [4] }\end{array}$ & 11 & $80 \%$ \\
$\begin{array}{l}\text { Euclidean } \\
\text { distance }\end{array}$ & $\begin{array}{c}\text { Nakjima } \text { et } \\
\text { al. [3] }\end{array}$ & 100 & 92.375 \\
$\begin{array}{l}\text { Ordinal Code } \\
\text { Competitive }\end{array}$ & $\begin{array}{c}\text { Wei Jia, } \text { et al. } \\
\text { [12] }\end{array}$ & 101 & $95 \%$ \\
$\begin{array}{l}\text { Code } \\
\text { BOCV }\end{array}$ & [5] & & 98.28 \\
\hline
\end{tabular}

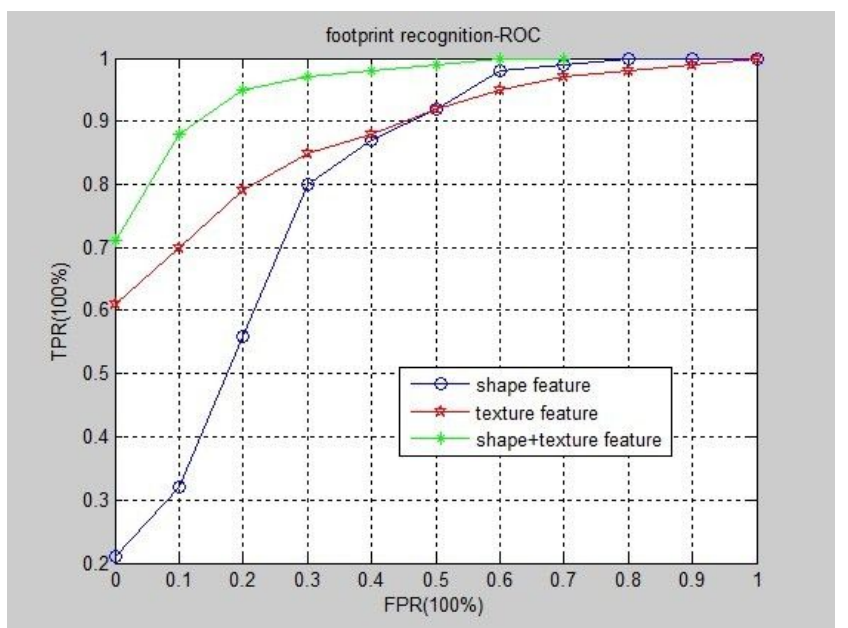

Figure 5: ROC of the Verification Results.

Figure 5 is the Receiver Operating Characteristic curve (ROC) of the verification results. The ROC curve is a visual rendition of the trade-off between the False Acceptance Rate (FAR) and the False reject Rate (FRR), in other words, the curve plots the true positives (sensitivity) against the false positives (specificity). False acceptance rate describes the chance that a biometric system will incorrectly authenticate a user or fail to detect an imposter. On the other hand, false reject rate describes the tendency where a system fails to authenticate an authorized user.

In general, the matching algorithm performs a decision based on a threshold which determines how close to a template the input needs to be for it to be considered a match. This curve presents a summary of the performance of our biometric verification system.

Biometric systems are fundamentally security systems with the primary objective of identifying the supposedly right people but sadly, recent researches have shown that an attacker can lift and replicate the biometric traits (fingerprints), which later can be used to launch an attack on biometric systems. These situations demanded that more imposture resistance biometric traits are given the needed attention than before. The study presents a biometric technique based on naked footprint, a technique that is largely obtrusive to reduce circumvention as much as possible and so capable of addressing the shortcomings of biometric traits that are easily susceptible to spoof 
attacks. It is therefore prudent to profess that footprint biometric must be employed to gain access to high security outfits as it is almost impossible to capture a person's footprint without his knowledge.

The study used two key features, shape and minutiae respectively from the single feature- naked footprint to give the system a multimodal effect at the speed of a unimodal system. Multimodal biometric systems which combine two different biometric modalities to verify a person's identity are a means of improving the accuracy of a biometric system.

As very diminutive literature is available for the footprint recognition systems, the study also seek contribute to the current study that is ongoing with regards active biometric traits in general and foot biometrics in particular. It is also to further enhance the assertion that footprint biometric is credible and is capable being developed to the level of other notable biometric features like face, iris, fingerprint etc. The results of the study make a strong case for an active biometric feature in naked footprint.

\section{CONCLUSION}

This paper presented a novel personal identification method using static footprint features viz. friction ridge and foot shape / silhouette. Verification accuracy is in the range of $98-99 \%$. Results show that the naked footprint is a credible biometric feature as two barefoot impressions of an individual match perfectly whilst that of two different persons shows a great deal of dissimilarity. We are of the view that Footprint biometrics though still in its early stages has the potential of dealing effectively with spoof attacks in biometric systems since its data acquisition is highly obtrusive.

\section{ACKNOWLEDGMENT}

This paper is supported by the National Natural Science Foundation of China (No. 61261029), and Jinchuan Company Research Foundation (JCYY 2013009).

\section{REFERENCES}

[1]. Ambeth, K. V. D. and Ramakrishnan M. (2010), Footprint recognition using modified sequential haar energy transform (MSHET), INTERNATIONAL JOURNAL OF COMPUTER SCIENCE ISSUES, (IJCSI) (7): 47

DOI: http://ijcsi.org/papers/7-3-5-47-51.pdf
[2]. Ambeth, K. V. D. and Ramakrishnan M. (2012), Footprint recognition with cop using principle component analysis (PCA), JOURNAL OF COMPUTATIONAL INFORMATION SYSTEMS (8): 4939-4950.

DOI:http://www.jofcis.com/publishedpapers/2012_8_12_4939_49 50.pdf

[3]. Chenyang, X. and Prince, J. L. (1998), Generalized gradient vector flow external forces for active contours, SIGNAL PROCESSING, (71), 131-139.

DOI:http://iacl.ece.jhu.edu/ chenyang/research/pubs/spij98.pdf

[4]. Hawes, M. R., Sovak, D., Miyashita, M., Kang, S. J., Yoshihuku, Y., Tanaka, S. (1994), Ethnic differences in forefoot shape and the determination of shoe comfort, ERGONOMICS, (37): 187. DOI:http://www.tandfonline.com/doi/abs/10.1080/0014013940 8963637?journalCode=terg20

[5]. Jung, J.W., Bien, Z., Lee S. W, Sato T., (2003), D Dynamic footprint based person Identification using mat-type pressure sensor, PROCEEDINGS OF THE 25 $5^{\mathrm{TH}}$ ANNUAL INTERNATIONAL CONFERENCE OF THE IEEE EMBS, Cancun Mexico, 2938-2940. DOI:http://ieeexplore.ieee.org/stamp/stamp.jsp?arnumber=01280 533

[6]. Marcialis G.L., Roli F., (2007), Score-level fusion of fingerprint and face matchers for personal verification under "stress" conditions, 14th INTERNATIONAL CONFERENCE ON IMAGE ANALYSIS AND PROCESSING, 259-264.

DOI:http://ieeexplore.ieee.org/xpls/abs_all.jsp?arnumber $=436278$ 9

[7]. Nakajima, K., Mizukami, Y., Tanaka, K., Tamura, T. (2000), Footprint based personal recognition, IEEE'S TRANSACTIONS ON BIOMEDICAL ENGINEERING, (47): 1534-1537.

DOI:http://ieeexplore.ieee.org/xpls/abs_all.jsp?arnumber=880106

[8]. Tunkpien, P., Phimoltares, S., Panthuwadeethorn S., (2011), Palmprint identification system using shape matching and KNearest neighbour algorithm, INSTITUTE OF ELECTRICAL AND ELECTRONIC ENGINEER'S INTERNATIONAL CONFERENCE ON IMAGING SYSTEMS AND TECHNIQUES, IST 2011, 327-330, DOI:http://ieeexplore.ieee.org/xpls/abs_all.jsp?arnumber=596222 7

[9]. Ravi, J.K., Raja, B., Venugopal, K. R (2009), Fingerprint recognition using minutia score matching, INTERNATIONAL JOURNAL OF ENGINEERING SCIENCE AND TECHNOLOGY. (1): 35-42, DOI:http://arxiv.org/ftp/arxiv/papers/1001/1001.4186.pdf

[10]. Ross A. A, Karthik N., Jain A. K, (2006), Handbook of multi biometrics, INTERNATIONAL SERIES ON BIOMETRICS, SPRINGER SCIENCE+BUSINESS MEDIA, LLC, 114 - 115 , ISBN-13: 978-0-38722296-7

[11]. Sébastien, M., Trusted biometrics under spoofing attacks ,TABULA RASA. DOI: http://www.tabularasa-euproject.org/

[12]. Wei, J., Hai-Yang, C., Jie, G., Rong-Xiang, H., Ying-Ke, L., Xiao-Feng, W. (2012), Newborn footprint recognition using orientation feature, NEURAL COMPUTING AND APPLICATIONS, (21): 1855-1863. DOI:http://link.springer.com/content/pdf/10.1007\%2Fs00521011-0530-9.pdf 